\title{
A Heuristic Non Destructive Evaluation Technique For Rebar Corrosion of Marine structures based on the synergy of Infrared Thermography, Signal and Image Processing.
}

\author{
byG. Kannan*, N. Manoharan* and B. Venkataraman ${ }^{* *}$ \\ *AMET University, Chennai, India \\ ${ }^{* *}$ IGCAR, Kalpakkam, Chennai, India
}

\begin{abstract}
The marine structures predominantly make Rebar as the reinforcement steel as tension device mainly to strengthen and hold concrete intact. As these structures are in constant contact with sea water, they develop corrosion due to salinity of water. There are emerging challenges in exact quantification of these parameters. The present paper aims to dwell on corrosion mechanisms including rebar corrosion and reviews the various evaluation techniques on thermography by elaborating the methods.A simplistic heuristic infrared thermography method is described for collecting images of the specimens and applying digital signal \& image processing techniques on them. Iterative data capturing is done for effective synergy of thermography and signal \& image processing techniques.
\end{abstract}

Keywords: Rebar Corrosion, marine structures, infrared thermography, signal and image processing, synergy, iterative methods.

\section{Introduction}

\subsection{Corrosion:}

Corrosion is the deterioration of a metal as a result of chemical reactions between it and the surrounding environment. Both the type of metal and the environmental conditions, particularly what gases that are in contact with the metal, determine the form and rate of deterioration. There are a numerous ways of addressing the 'corrosion' and are described as 'General Attack corrosion', 'localised corrosion', Galvanic Corrosion, Environmental cracking, flow assisted corrosion, de-alloying, fretting corrosion, High-Temperature Corrosion.

Corrosion of steel in concrete Reinforced concrete structures perform well as long as the alkaline environment is intact [3].

The main reasons for depassivating concrete are carbonation of concrete and chloride penetration. Carbonation induced corrosion is a problem where concrete cover is small or the concrete of bad quality with well connected open pores and a low cement ratio with poor corrosion of reinforcement has been established as the predominant factor causing widespread premature deterioration of concrete construction worldwide, especially of the structures located in the coastal marine environment [10]. The most important causes of corrosion initiation of reinforcing steel are the ingress of chloride ions and carbon dioxide to the steel surface. After initiation of the corrosion process, the corrosion products (iron oxides and hydroxides) are usually deposited in the restricted space in the concrete around the steel. Their formation within this restricted space sets up expansive stresses, which crack and spall the concrete cover. This in turn results in progressive deterioration of the concrete. As a result, the repair costs nowadays constitute a major part of the current spending on for measurement of the corrosion rate of reinforcing steel in concrete, many electrochemical and non-destructive techniques are available for monitoring corrosion of steel in concrete structures. Rebar corrosion on existing structures can be assessed by different methods such as, Open circuit potential (OCP) measurements, galvanostatic pulse transient method, electrochemical spectroscopy, ultrasonic and X-ray measurements. In Open Circuit Potential (OCP) Measurements, the tendency of any metal to react with an environment is indicated by the potential it develops in contact with the environment.

\subsection{Rebar Corrosion:}

In reinforced concrete structures, concrete acts, as an electrolyte and manifestation of corrosion of steel in concrete Rebar is explained in the following manner. The rebar in marine concrete structures perform as desired provided the alkaline environment is not disturbed and is kept intact. When the passivating effect of the concrete with $\mathrm{pH} 10-12$ is penetrated, corrosion of steel in concrete can occur. Chloride ion, present in marine breeze and seawater is considered the main external agent to damage reinforced concrete in marine environments. It affects the passivity of steel film and provokes the initiation of corrosion. Concrete is a very important construction material [4]. It is highly resistant to compression forces, but week under traction forces. To improve its properties, it is combined with steel bars, highly resistant to traction. Concrete offers corrosion protection to carbon steel. It acts like a physical barrier that partially isolates steel surface from the external environment and establishes an alkaline $\mathrm{pH}$ that facilitates steel passivity [5]. Durability of a reinforced concrete structure depends on the 
environment in which it is exposed, as also on the time and properties of concrete. Permeability is an important property in determining sensibility of concrete to external factors. For high durability, concrete should have low permeability that is strongly linked to porosity of the concrete paste.

Carbonization and chloride penetration effect are the predominant contributing factors in corrosion[1]. Innumerous structures are contaminated with chloride as a consequence of deicing during winter and chloride laden environment such as marine offshore and coastal structures. The marine structures are categorized, by one school of thought, into five major categories namely i) piled platforms ii) flexible bulk heads iii) gravity structures iv) rubble mounds and v) floating structures. These structures predominantly make Rebar as the reinforcement steel as tension device mainly to strengthen and hold the concrete intact. These structures are contact with sea water and hence are subjected to corrosion as described earlier and may be described as the deterioration of materials especially metals by chemical or electrochemical reaction with environment. Though it is simply stated, the exact quantification of the corrosion parameters, their measurements, and their characterization with time are all real challenging tasks [8]. Various Non Destructive Evaluation techniques using conventional methods of radiography, ultrasonic, thermography and the like are deployed these purposes [2].

It is the endeavor of this paper to review various Non-destructive techniques and variants of thermography methods. Different methodology and signal processing techniques will be studied from the point of efficacy and merits and demerits on the existing conventional methods. The lock-in thermographic methods will be studied elaborately and how this method is best suited for defect characterization and quantification for rebar corrosion.

\subsection{Various Evaluation techniques in thermography:}

There exist several types of thermography approaches which can be classifiedbased of the methodology for testing the materials, the origin of the source of thermalenergy and the measured temperature differences arising from it. In general; the infraredthermography IRT techniques can be classified into two main types .

Passive, steady state or static thermography; this technique operates in two

wavelength ranges, 3-5 $\mu \mathrm{m}$ (SWIR) and 8-12 $\mu \mathrm{m}$ (LWIR) [6].Where IR radiationenergy depends on the chosen wavelength range. Passive thermography isessentially based on analysis where no external excitation is used to excitethermal gradients on the test specimen's surface[11]. In this approach, thefeatures of interest are naturally at a higher or lower temperature than thebackground. Passive thermography has many applications such as surveillanceof people on a scene and medical diagnosis; specifically thermology)[12]

Active or dynamic thermography; this technique is based on the detectionand recording by an infrared camera of thermal IR radiations emitted byobject surface. External energy source is required to be used in thisapproach in order to produce a thermal contrast between the examinedspecimen and the feature of interest (subsurface defects); where it isrequired to heat or cool the object being inspected in order to create therequired thermal contrast[11]. The active approach is necessary in manycases given that the parts being examined are usually in equilibrium withthe surroundings[12].

Infrared Thermography (IRT) is one of the most attractive nondestructiveinspection NDI methods because it is a non-contact technique, safe, clean, painless, andsuch a fast inspection method and is used as an NDT technique in variety of applications from medical to building inspections. that and is becoming a widely adopted technique to detectthe presence of variety of internal flaws or defects; i.e. discontinuity or nonhomogeneity inside materials

IRT has many advantages like they are non-contact technique, can create 2D thermal maps (thermogram), they are direct tests in real time and can reduce maintenance costs and finally with no harmful radiation effects.

Toobtain maximum information about the corrosion state of rebar in a particular structure, acombination of measuring techniques is recommended. The electrochemical corrosionmeasurements are usually qualitative and also semi quantitative; significant benefits can bederived from them. The development of durable, embeddable sensors and inexpensive microprocessor control andcommunications, have encouraged the development of corrosion monitoring systems for newand existing reinforced concrete structures [7]. The development of integrated monitoring systemsfor new and existing reinforced concrete structures could reduce costs by allowing a morerational approach to the assessment of concrete structures, the ability to continuously monitor the cover concrete and steel in real time could thus able toprovide more information of the current and future performance of the structure;.

The minimum number of experiment components needed to perform infrared thermography are heat source and an infrared (IR) camera. The proper choice of heat source is paramount for a successful thermographic experiment. Such source must be chosen so as to maximize the thermal contrast between the defect and the surrounding material.

Any material can be inspected thermographically (such as metals, ceramics and composites) but the outcome of the inspection will depend on many factors such as sample geometry, sample thickness, amount of heat used, surface emissivity, specific heat, material density, thermal conductivity, defect depth and the size of the defect and other parameters. 
The infrared temperature measurements are affected bymany of the same factors including surface emittance, air temperature, backgroundthermal radiation, and air humidity

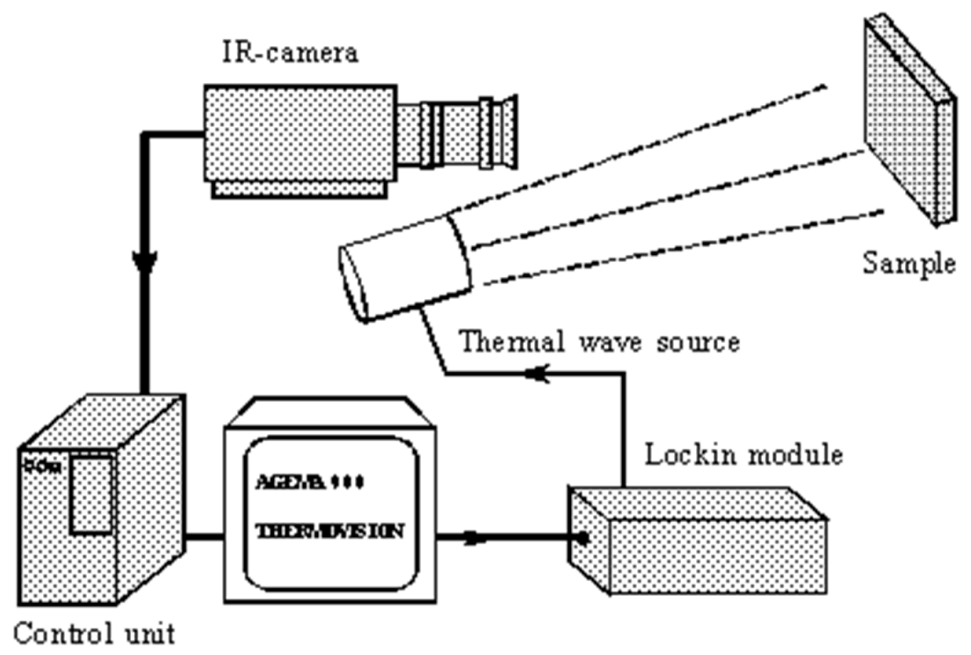

Figure 1 Schematic of Lock-In thermography Technique

\section{Lock-in thermography technique (LT)}

Lock-in Thermography stands as an active thermography NDI technique which isestablished basing on using a periodic input energy waves (periodic excitation) to sendperiodic waves (sinusoids) at a given modulation frequency $\omega$; in order to deriveinformation from the observed phase and amplitude of the reflected thermal wave[13]. The halogen lamps represent the most common affordable stimulationheating source that is used in LT[14].

Lock-in thermography is a technique derived from photo-thermal radiometry(PTR)[15], in which, the thermal waves is delivered into specimen's surface in the formof periodic thermal waves (periodic stimulation), then the thermal response is recorded atthe same time by using an IR camera[12,16]. In general in PTR, the examinedspecimen's surface is stimulated by a plane light beam and its thermal infrared emissionis measured and recorded by an infrared IR detector. In one hand, if the infrared IRdetector is made of a monolithic IR sensor, then the technique is known as photo-thermalradiometry (PTR), whereas if the detector is made of an array of IR sensors, thetechnique is known as lock-in thermography $(\mathrm{LT})[13,17]$. Using an un-cooled IRcamerain $L T$ is normally sufficient, and then it is an economical technique since theuncooled IR camera is cheaper.

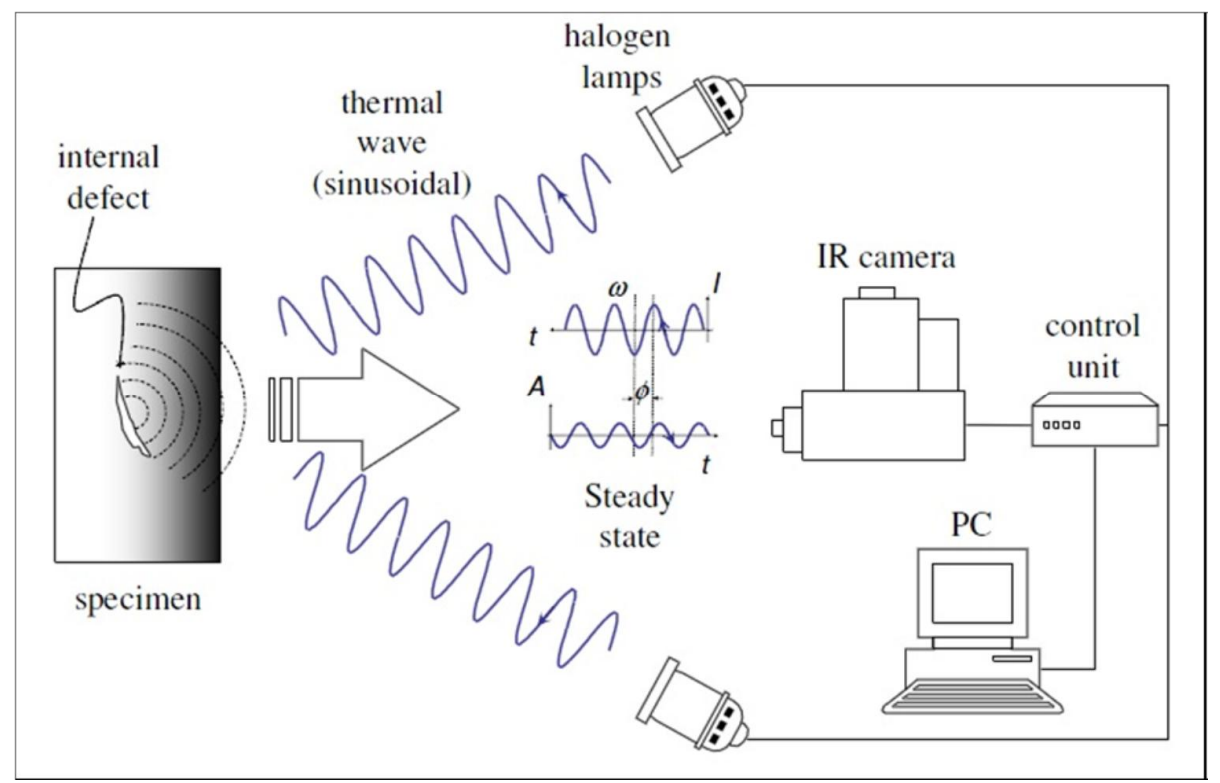

Figure 2: Equipment configurations for Lock-in Thermography 


\subsection{Principle:}

The principle of LT operation is based on applying periodic thermal waves into

the specimen being inspected. The periodic wave propagates by radiation through the airuntil it reaches the examined object's surface where heat energy is generated and warmthe specimen's surface up then propagates by diffusion through the material as thermalwaves. Whenever these thermal waves reach any discontinuity (defect); such asinclusions, or delaminations, then these defect acts as a thermal barrier (thermalresistance) causing changes in amplitude and phase delay (shifting) of the response signalat the surface. The thermal response is recorded at the same time by using an IR camera[16,18,19]. LT technique has established basing on the fact that the thermal wave is veryresponded to any slight changes or interfaces between materials, where based onobserved thermal images that obtained via IR camera, then any changes in amplitude orphase between defective areas and non-defective areas refers to the defect's presence. In LT technique, it is preferred to use halogen lamps to generate the periodicthermal waves, due to their relatively high efficiency, simplicity in use and possibility ofcontrol by amplitude modulation of conventional power units[13].

The most interesting thing as a NDI technique is these periodic thermal waves canbe created and detected remotely. In general; lock-in thermography is convenient tool tobe used for detecting subsurface defects, measuring thicknesses of coatings anddetermining material properties [9]. Basically, the lock-in terminology refers to the fact of being required to monitorthe exact time dependence between the output signal and the original input signal.

Qualitatively, the phenomenon is as follows; in this technique the examinedspecimen's surface is periodically stimulated by one or several modulated heatingsources, such as halogen lamps, to inject thermal waves perpendicularly into theinspected specimen. Where the periodic thermal waves transfer by radiation through theair (medium) until the sinusoidal heat wave's front being in face with the inspectedspecimen's surface where the inspected specimen's surface is heating up then the thermalwaves propagates by diffusion through the material. The temperature of each point in theinspected specimen will change over time, as it will be affected by the generated wavesand those reflected in thermal barriers. Since the subsurface defect or any subsurfacediscontinuity or non-homogeneity act as barrier for heat propagation, then it will effect onthe thermal waves and change its amplitude and phase as a result.

Simply, wherever these thermal waves being in face with any subsurfacediscontinuity or nonhomogeneity in which there is a change of the thermal physicalproperties, causing a thermal effect change in terms of reducing the amplitude and itsphase delay, then it will be partially reflected. The reflected part of the thermal waveinterferes with the incoming input thermal wave creating at the stimulated surface of theexamined specimen, causing an interference pattern in the local surface temperature andthus in the surface radiation as a result; which oscillates at the same frequency as theinput thermal wave. The thermal response is recorded at the same time by using an IRcamera, then evaluating the amplitude and the phase of the local surface temperatures oneto earn information about the internal structure of test object. The IR camera can observethe whole or a large part of the inspected specimen's surface typically in a $320 \times 256$ or640 × 512 pixel array configurations $[20,12,21,16,22]$.

Any phase shift (delay) between the thermal evolution for a defective region and anon-defective region represents an abnormality (defect) which can be observed andrevealed. For NDI testing purposes, the phase image (phasegram) is often moreinformative than the ampligram (amplitude image) one which strongly depends on thelocal IR emissivity; i.e. the phasegram is independent from the IR emissivity $(\varepsilon)$ of thesurface. In fact, the phasegram is a measure of the time delay of the surface temperaturemodulation referred to the power modulation, which is indeed independent of themagnitude of the heat source[23].

Mathematically, in the lock-in thermography technique the procedure as follow after observing the inspected specimen's surface via IR camera then the recordedtemperature data is transformed into a frequency domain. In which in each pixel, themeasured temporal evolution of the temperature represents a Fouriertransformed for allthe recorded images sequence. Each pixel in the thermal image corresponds to atemperature at a given time, so that by using Fourier-Transform method for each pixelper cycle then an advanced new result is obtained. This new result is represented by aphase image and an amplitude image. Any fault (discontinuity or nonhomogeneity)inside the object will be reflected in these images; hence they can show theinternal structure of the inspected object and all possible defects.

\subsection{Experimental Details:}

The experimental configuration of lock-inthermography technique includes a periodic thermal stimulation source in order to deliverthe thermal energy to the inspected specimen's surface in form of periodic thermal. Thehalogen lamps send periodic thermal waves to the inspected specimen's surface at agiven modulation frequency $\omega$, for at least one cycle; ideally until reaching a steady state,which depends on the thermal physical properties of the examined specimen and the defect depth. The thermal response is recorded at the same time using an IRcamera that is synchronized with the excitation signal and decomposed by a lock-inamplifier to extract the amplitude and phase of the modulation.

In LT, the object beingexamined is periodically excited by using a periodic thermal wave with a singlefrequency corresponding to a particular depth at which a particular defect can bedetectable. However; this procedure (test) has to be repeated with other differentfrequencies to cover a wide range of depths (each single frequency to detect a defectpresence at specific test depth).

The data acquisitions in LT technique are more accurate depending onthe phasegram of the output signal. The lock-in thermography LT, allows the reconstruction of phaseimages that are less affected by the 
characteristics mentioned above (non-uniformheating, emissivity variations, surface geometry, and environmental reflections); i.e. LTrepresents an insensitive technique[25].

An interesting thing of LT is in the fact that by the use of a periodic excitationdetailed examinations can be executed with a relatively low power thermal waves inputinto the object. This permits the examination of thermally sensitive components and theuse of relatively simple heat sources.

\subsection{Data Analysis}

Preparing a building component specimen for thermography is somewhat similar to hotbox testing, with differences in flush mounting and emittance modifications. Large areaspecimens can simply be located between the chambers with perimeter insulation distances can be measured for subsequent synchronization with thethermographic data. This step is critical to obtaining accurate spatial coordinates to pairwith infrared temperature data.

The infrared imaging system should be positioned as close to the specimen as is practical,allowing the imager to view the desired portion of the specimen, the reference emitter, and at least two location markers, and also allowing the background mirror to bedeployed. Each thermogram should include enough of the reference emitter to provide atleast 10 horizontal and 10 vertical infrared elements or IFOVs. How much of thespecimen is selected for viewing will vary depending on the specimen's geometry andthermal contrast; small features and high surface temperature gradients require closeviewing. The maximum viewing distance to resolve a given feature is determined by thelFOV of the infrared imaging system where three to five (or more) IFOVs are needed toresolve the temperature of a feature. The temperature span of the infrared imaging systemshould be the smallest possible value that includes all the features of interest. Thereference emitter surface temperature should be set to within $\pm 3^{\circ} \mathrm{C}$ of the temperature of agiven feature of interest. Thermograms should be recorded by averaging data over time, usually by averaging multiple frames of data. For analog systems, 20 frames or more isrecommended. Depending on the range of temperatures present it may be necessary torecord several thermograms with smaller temperature spans and varying centertemperatures. When multiple images with different spans and center temperatures aretaken of the same view, good data alignment can be insured by including at the time ofcapture geometry overlays that will be used for postprocessing in all the relatedthermograms. Immediately before and/or after imaging a particular view of the specimen, deploy the reflective background mirror parallel to and in front of the test specimen andrecord the effective background radiation level (emittance set to 1.0). The imager viewingangle is usually at an offset from normal to prevent reflections of the cold scanner lens, which is an unavoidable nonuniformity in the background.

The specimens with introduction of various defects were manufactured and subjected to saline environment. The corrosion effects are studied in details;

\subsection{Merits and demerits of IRLT Techniques}

Infrared Thermography is an attractive and powerful nondestructive testing and evaluating inspection technique that provides a safe remote (non-contact) inspection of components and structures without changing the integrity and properties of the examined specimens and without causing any damage, through a mapping of thermal patterns on the surface of the objects of interest. Defect detection fundamental in active

thermography is based on the fact that the variations in temperature that exists between the reference area and a defective region based on its emitted infrared radiations, which can be used for defect detection and quantification purposes.

\section{Conclusion:}

The results of Lock-in thermographic data are acquired by preparing the experimental set up and also by conventional techniques. The initial interpretation of results are encouraging that the thermography methods for rebar corrosion specimens will turn out to be more effective and in-situ methods can be conceived for field applications

\section{References:}

1.Ha-Won Song, VeluSaraswathy "Corrosion Monitoring of Reinforced Concrete Structures - A Review" Int. J. Electrochem. Sci., 2 (2007) 1- 28

2."Non Destructive Assessment Of Concrete Structures: Reliability And Limits Of Single And Combined Techniques" 2012 ISBN 978-94-007-2735-9

3.Alan D. Zdunek and David Prine "Early Detection of Steel Rebar Corrosion by Acoustic EmissionMonitoring" Paper No. 547

4.T. paulTeng "material and methods for corrosion control of reinforced and prestresses concrete structures in new construction " P.No 00-081, Aug 2000

5.Ralf ARNDT, Frank JALINOOS "NDE for corrosion detection in reinforced concrete structures - abenchmark approach" June 30th - July 3rd, 2009 
6.Yuhua Cheng, Yiming Deng, Jing Cao, XinXiong, LibingBai and Zhanjun Li - Review on "Multi-Wave and Hybrid Imaging Techniques: A New Direction for Nondestructive Testing and Structural Health Monitoring Sensors 2013, 13, 16146-16190 doi: 10.3390/s131216146

7.Sanjeev Kumar Verma, Sudhir Singh Bhadauria and SaleemAkhtar - Review Article "Review of Nondestructive Testing Methods for Condition Monitoring of Concrete Structures - Journal of Construction Engineering Accepted in 13 March 2013

8.The Effects and Economic Impact of Corrosion

9.Lock-in Thermography textbook, Basics and Use for Evaluating ElectronicDevices and Materials, Second Edition, O. Breitenstein, W. Warta, M.Langenkamp, May 2010.

10. M. Sosa1, T. Pérez-López1, ${ }^{*}$, J. Reyes1, F. Corvo1, R. Camacho-Chab2, P. Quintana3, D. Aguilar.Int. J. Electrochem. Sci., 6 (2011) 6300 - 6318 Influence of the Marine Environment on Reinforced Concrete Degradation Depending on Exposure Conditions

11. Maldague, "Introduction to NDT by Active Infrared Thermography", MaterialsEvaluation, Volume 60, Issue 9, 2002, Pages 1-22.

12. Maldague X. P. V., Streckert H. H and Trimm M. W. "Introduction to infraredand thermal testing: Part 1. Nondestructive testing," in Nondestructive Handbook, Infrared and Thermal Testing, Volume 3, X. Maldague technical ed., P. O. Mooreed., 3rd edition, Columbus, Ohio, ASNT Press, 2001, 718 p.151

13. Thermographic NDT System, AnnaVladovaAndonova and DimitarGeorgievTodorov

14. NDT in Composite Materials with Flash, Transient, and Lockin Thermography,Markus Tarin and Ralph RotolanteMoviTHERM, Inc.

15. Nordal P. E. and Kanstand S. O. "Photothermal radiometry," PhysicaScripta,20:659-662, 1979.

16. Active infrared thermography techniques for the nondestructive testing ofmaterials, Clemente IbarraCastanedo, Marc Genest, Jean-Marc Piau, StéphaneGuibert, AbdelhakimBendada and Xavier P. V. Maldague 17. Simultaneous measurement of thermal diffusivity and optical absorptioncoefficient using photothermal radiometry. I. Homogeneous solids, RaquelFuente, EstibalizApiñaniz, ArantzaMendioroz, and Agustı'n Salazar 18. An IR Lock-in Thermography Nondestructive Test System Based on the ImageSequence Processing, Junyan LIU , Jingmin DAI , Yang WANG , Hui LIU andZijun WANG

19. Introduction to NDT by Active Infrared Thermography, X. Maldague

20. X.P.V. Maldague, Theory and Practice of Infrared Technology for NondestructiveTesting, Wiley, New York (2001)

21. Inspection of aerospace materials by pulsed thermography, lock-in thermographyand vibrothermography: A comparative study, Clemente Ibarra-Castanedo, MarcGenest, StéphaneGuibert, Jean-Marc Piau, Xavier P. V. Maldague andAbdelhakimBendada.

22. Quantitative subsurface defect evaluation by pulsed phase thermography: depthretrieval with the phase, Clemente Ibarra Castanedo, 2005

23. Thermal Failure Analysis by IR Lock-in Thermography, O. Breitenstein, C.Schmidt, F. Altmann, D. Karg

24. Numerical Modeling of Infrared Thermography techniques via ANSYS

25. Quantitative subsurface defect evaluation by pulsed phase thermography: depthretrieval with the phase, Clemente Ibarra Castanedo, 2005. 